\title{
ERRATUM
}

Peter Ván • Arkadi Berezovski • Christina Papenfuss

\section{Erratum to: Thermodynamic approach to generalized continua}

Published online: 24 January 2014

(C) Springer-Verlag Berlin Heidelberg 2014

\section{Erratum to: Continuum Mech. Thermodyn. (2013) 118 DOI 10.1007/s00161-013-0311-z}

The evolution equations for the internal variables $\psi_{i j}$ and $\beta_{i j}$ in formula (5) on p. 4 are mistyped in the published article. The correct expressions are

$$
\dot{\psi}_{i j}+f_{i j}=0, \quad \dot{\beta}_{i j}+g_{i j}=0 .
$$

There are some consequent sign mistakes in the following.

On p. 4 Eq. (9) for the derivative constraints and Eq. (10) for the entropy imbalance should be corrected as

$$
\partial_{k} \dot{\psi}_{i j}+\partial_{k} f_{i j}=0, \quad \partial_{k} \dot{\beta}_{i j}+\partial_{k} g_{i j}=0,
$$

and

$$
\begin{aligned}
& \rho_{0} \dot{s}+\partial_{j} J_{j}-\Lambda_{j i}\left(\dot{F}_{i j}-\partial_{j} v_{i}\right)-\lambda_{i}\left(\rho_{0} \dot{v}_{i}-\partial_{j} t_{i j}\right)-\kappa\left(\rho_{0} \dot{e}+\partial_{i} q_{i}-t_{i j} \partial_{j} v_{i}\right) \\
& +A_{j i}\left(\dot{\psi}_{i j}+f_{i j}\right)+\mathscr{A}_{k j i}\left(\partial_{k} \dot{\psi}_{i j}+\partial_{k} f_{i j}\right) \\
& +B_{j i}\left(\dot{\beta}_{i j}+g_{i j}\right)+\mathscr{B}_{k j i}\left(\partial_{k} \dot{\beta}_{i j}+\partial_{k} g_{i j}\right) \geq 0 .
\end{aligned}
$$

The online version of the original article can be found under doi:10.1007/s00161-013-0311-z.

P. Ván $(\varangle)$

Department of Theoretical Physics, Wigner RCP, HAS, Konkoly Thege Miklós út 29-33., 1121 Budapest, Hungary

E-mail: van.peter@wigner.mta.hu

Tel.: +36-1-3922222

Fax: +36-1-3922727

P. Ván

Department of Energy Engineering, Budapest University of Technology and Economics, Bertalan Lajos u. 4-6,

1111 Budapest, Hungary

P. Ván

Montavid Thermodynamic Research Group, Budapest, Hungary

A. Berezovski

Centre for Nonlinear Studies, Institute of Cybernetics, Tallinn University of Technology, Akadeemia tee 21,

12618 Tallinn, Estonia

C. Papenfuss

Technical University of Berlin, Strasse des 17. Juni 135, 10623 Berlin, Germany 
On p. 8 Eq. (50) are written correctly as

$$
\stackrel{\diamond}{\psi}_{i j}+f_{i j}=0, \quad \stackrel{\diamond}{\beta}_{i j}+g_{i j}=0 .
$$

Further on the sign error appears on p. 11 in formula (69) for the entropy production of standard linear solid, which should be replaced by

$$
T \sigma=\left(t_{i j}-\rho \partial_{\varepsilon_{i j}} w\right) \dot{\varepsilon}_{i j}+\partial_{\psi_{i j}} w \rho f^{i j} \geq 0 .
$$

Finally, formula (86) on p. 13 of entropy production should be written as

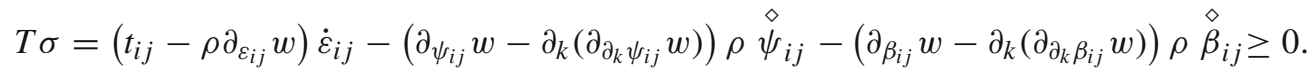

